\title{
Culturas de salud y encuentro clínico: percepción de los profesionales sobre las dimensiones que median en el encuentro terapéutico
}

\author{
Arantxa GRAU-MUÑOZ, David MUÑOZ-RODRÍGUEZ, Elisabet MARCO-AROCAS
}

Introducción. El encuentro clínico ha sido entendido como un encuentro entre culturas de salud mediado por dimensiones: el paciente como sujeto de conocimiento, el uso de otras fuentes de información, los procesos de negociación y la implicación de los aspectos culturales. El proyecto Explora 'Lenguaje y cultura de la salud' contemplaba la realización de una encuesta sobre las cuatro dimensiones atribuidas al encuentro clínico.

Sujetos y métodos. La población de estudio estuvo conformada por profesionales del ámbito de la salud en activo, que desarrollan su actividad profesional en centros de titularidad pública o privada. La muestra la componen 200 profesionales registrados en Revista de Neurología que aceptaron participar en el estudio, y cuya información fue posteriormente analizada por el equipo investigador mediante el programa SPSS v. 23.

Resultados y conclusiones. Los resultados obtenidos apuntan a la existencia de una posición mayoritaria escéptica con las disposiciones culturales de los pacientes, que se ve matizada por una afirmación positiva ante la necesidad de horizontalidad en el encuentro clínico.

Palabras clave. Autonomía del paciente. Competencia cultural. Comunicación. Culturas de salud. Encuentro clínico. Relación médico-paciente.

Cultures of health and clinical encounter: perception of the professionals on the dimensions that mediate in the therapeutic encounter

Introduction. The clinical encounter has been understood as an encounter between health cultures mediated by dimensions: the patient as a subject of knowledge, the use of other sources of information, the negotiation processes and the implication of cultural aspects. The Explora 'Language and culture of health' project contemplated carrying out a survey on the four dimensions attributed to the clinical encounter.

Subjects and methods. The study population consisted of professionals in the field of active health, who develop their professional activity in public or private ownership centers. The sample is composed of 200 professionals registered in Revista de Neurología who accepted to participate in the study, and whose information was later analyzed by the research team through the SPSS v. 23 software.

Results and conclusions. The results obtained point to the existence of a majority position skeptical with the cultural dispositions of the patients, which is qualified by a positive affirmation in view of the need for horizontality in the clinical encounter.

Key words. Autonomy of the patient. Clinical encounter. Communication. Cultural competence. Doctor-patient relationship. Health cultures.

\section{Introducción}

Talcott Parsons (1951) abordó la relación medicopaciente desde su propuesta funcionalista, entendiéndola como un juego diferenciado de roles en el que se reconocía al médico la posición activa de conocimiento en el encuentro clínico, mientras que al paciente se le reservaba un rol pasivo de asunción de las indicaciones del primero [1]. El paternalismo que se deriva de este abordaje, según Seoane [2], requiere al profesional asistencial -superior en conocimientos técnicos, experiencia y pericia- el favorecer los intereses del paciente o usuario, y hacerlo según la lectura de estos. Ello comporta la limitación de la autonomía o la libertad de decisión y acción de los pacientes. Sin embargo, las fisonomías de las sociedades actuales exigen un cambio de perspectiva que redefina el encuentro terapéutico, haciéndo-
Departamento de Sociología y Antropología Social. Universitat de València. Valencia, España.

Correspondencia: Dra. Arantxa Grau Muñoz. Departamento de Sociología y Antropología Social. Universitat de València. Avda. Blasco Ibáñez, 21. E-46010 Valencia.

E-mail: arantxa.grau@uv.es

Recibido: 25.09.18.

Aceptado: 27.09.18.

Conflicto de intereses: No declarado.

Competing interests: None declared.

(c) 2019 FEM 
lo transitar desde la relación clínica clásica a un modelo de relación asistencial de carácter sociosanitario. La relación clínica del siglo XXI, según Seoane, 'no es igual a la relación diádica marcada por el encuentro entre un médico y un enfermo, sino el resultado de la evolución y reinterpretación de aquella relación y la incorporación de nuevos elementos y nuevos personajes' [3]. Nos estamos refiriendo a lo que se ha denominado 'modelo de atención centrado en el paciente', que pretende superar el modelo asistencial focalizado en la enfermedad [4].

El modelo centrado en el paciente involucra a los usuarios en todos los aspectos de su cuidado. Los considera desde una mirada holística, atendiendo a sus necesidades físicas, psicológicas y sociales y, por lo tanto, transita desde la práctica clínica caracterizada por una mínima comunicación a una comunicación abierta [5]. La resolución de los problemas de salud sigue implicando la interacción entre dos sujetos (o más), y todas las funciones que inciden en la construcción de la relación clínica se cumplen mediante recursos discursivos [6].

Desde este marco de referencia, y queriendo atender a las relaciones profesional-usuario mediadas por recursos discursivos, se ha caracterizado el encuentro terapéutico a través de cuatro dimensiones, que se describen a continuación.

\section{Sujetos portadores de conocimiento propio}

Los profesionales sanitarios deben esforzarse por evitar actitudes que induzcan a los usuarios a sentirse objetos. Una actitud distante, en la que no se atiende el estado emocional ni las necesidades de información de quien consulta, puede llevar a que los pacientes se sientan cosificados [7]. Según la revisión bibliográfica de Cófreces et al, aunque existe cierto consenso en la literatura científica en que la interacción profesional-usuario es un fenómeno multicausal, también hay acuerdo en que niveles altos de adhesión al tratamiento se asocian con modelos de comunicación que tienen en cuenta las actitudes, las creencias y las preferencias de los pacientes y permiten un proceso de negociación con mayor acuerdo y comprensión [8].

\section{Convivencia con otras fuentes de información. Internet}

La disposición de información de calidad emerge como premisa para una buena participación por parte del usuario en la toma de decisiones sobre su propia salud. Sin embargo, diversos estudios demuestran que la información proporcionada por los profesionales no siempre es todo lo completa que debería e incluso que una parte de la población no consigue comprender totalmente lo que se le transmite [9]. Actualmente, internet se ha convertido en una fuente a la cual acuden los pacientes cuando tienen lagunas al respecto [10]. Sin embargo, según señalan algunos estudios, la predisposición del colectivo profesional sanitario ante la emergencia de este fenómeno no parece ser muy favorable, apuntando desventajas en relación al paciente, como el exceso o la falta de veracidad de la información o posibles desajustes entre la información proporcionada por el profesional y la de la red [11].

\section{Negociación en una toma de decisiones compartida y consensuada}

La promoción de la implicación de los pacientes en la toma de decisiones constituye una dimensión fundamental en la exigencia de compartir el poder y la responsabilidad que proponen la mayoría de las aproximaciones centradas en el paciente [12]. La corresponsabilidad que deben asumir profesionales y usuarios pasa necesariamente por el compromiso mutuo, pero también por el diálogo. Las decisiones compartidas exigen un diálogo que debe posibilitar la individualización de las intervenciones con los pacientes. Desde la perspectiva de la toma de decisiones en salud compartida, los profesionales deben ofrecer información sobre las opciones de tratamiento, obtener información sobre las preferencias del paciente y acordar los siguientes pasos para la toma de decisiones [13].

\section{Competencia cultural}

La bibliografía apunta que los pacientes de grupos minoritarios, especialmente con un dominio limitado de la lengua mayoritaria, están más expuestos a no obtener una respuesta empática por parte de los profesionales, a recibir una información más pobre por parte de estos, y a no ser alentados a la participación activa en la toma de decisiones [14]. Sin embargo, la referencia al uso de lenguas distintas en el encuentro clínico no resulta suficiente para explicar los choques culturales que se producen entre profesionales y usuarios. Hablamos de una prestación de cuidados de salud con competencia cultural como la atención que da respuesta a la diversidad de la población y, por lo tanto, a los factores culturales que pueden afectar a la salud y a la atención sanitaria, como el idioma, los estilos de comunicación, las creencias, las actitudes y los comportamientos. Para ser culturalmente competentes, los proveedores de atención de la salud tienen que em- 
plear diversas estrategias interpersonales y organizativas para superar las barreras de comunicación y comprensión que se derivan de las diferencias raciales, étnicas, culturales y lingüísticas [15].

Desde la perspectiva que asumimos en esta investigación, la postura de los profesionales de la salud ante estas cuatro dimensiones permitirá dibujar su posicionamiento ante procesos de cesión de poder y, por lo tanto, su cercanía a unas relaciones profesional-usuario que den respuesta al modelo asistencial centrado en el paciente.

El presente artículo recoge parte de los resultados del proyecto Explora 'Lenguaje y cultura de la salud' (CSO2014-61928-EXP). El proyecto tenía como objetivo principal analizar las representaciones de los profesionales de la salud en relación al encuentro clínico, contemplando los aspectos relativos a las percepciones sobre las interacciones producidas en dicho encuentro y aquellos aspectos referidos a la percepción que los profesionales tienen sobre los pacientes en tanto que sujetos portadores de nociones y conocimientos referidos a su propia salud.

Con el fin de conseguir este objetivo se establecieron cuatro objetivos específicos, referido cada uno de ellos a una dimensión considerada relevante en la configuración del encuentro terapéutico:

- Identificar cómo consideran los profesionales a los pacientes en tanto que sujetos portadores de un conocimiento propio.

- Analizar las percepciones de los profesionales sobre el uso de las nuevas tecnologías de la información (internet) por parte de los pacientes.

- Conocer las representaciones sobre el encuentro clínico como espacio en el que cabe cierta negociación con los pacientes.

- Analizar las percepciones sobre los aspectos culturales de los pacientes que pueden influir en las nociones de salud de estos últimos.

\section{Sujetos y métodos}

El proyecto contempla la triangulación metodológica como estrategia para profundizar en la obtención de resultados. Así, los resultados de la fase cualitativa constituyen la base para la elaboración del cuestionario utilizado en la fase cuantitativa. A partir de estos aspectos, se construyeron las dimensiones a través de las cuales se hicieron operativos los cuatro objetivos específicos y que estructuraron el cuestionario.

La población de estudio está conformada por profesionales en activo del ámbito de la salud, per- tenecientes a categorías sanitarias medias y superiores, que desarrollan su actividad profesional en centros de titularidad pública y privada.

En el diseño muestral se tuvo en cuenta la posibilidad de acceso a la población y el tiempo estimado para la distribución del cuestionario. Además, se consideró también que la población objeto de estudio está compuesta por perfiles de profesionales de un campo específico. Con estas consideraciones se optó por un diseño muestral intencional no probabilístico. La muestra intencional se conforma en función de criterios o restricciones no aleatorias, como las enunciadas, permitiendo llegar rápidamente a una muestra objetivo y en las que prescindir del muestreo por proporcionalidad no resta significación a los resultados.

Así, la muestra estuvo compuesta por aquellas personas que tenían las características descritas: ser profesional en activo perteneciente a una de las categorías sanitarias consideradas: médico, farmacéutico/a, psicólogo/a, titulado/a superior con función sanitaria (biología, física, química), enfermero/a, matrón/a, fisioterapeuta, técnico/a sanitario/a (radiodiagnóstico, laboratorio, medicina nuclear, radioterapia, dietética y nutrición, y anatomía patológica), u otra categoría sanitaria distinta de las anteriores. No se estableció afijación proporcional para las distintas categorías sanitarias.

La validez de una muestra no probabilística no reside tanto en la representatividad de sus elementos, sino en la elección de sujetos con ciertas características definidas previamente en el planteamiento del problema y que son relevantes en el campo de estudio. Por tanto, para muestras no probabilísticas, no procede estimar el intervalo de confianza ni el margen de error. En este sentido, en el presente proyecto se valoró la omisión de la estimación de estadísticos vinculados con el tamaño muestral. No obstante, después de realizar varios cálculos para muestras probabilísticas hipotéticas, se decidió alcanzar 200 cuestionarios individuales.

En lo referente a las estrategias para la distribución del cuestionario, se decidió optar por un cuestionario autocumplimentado mediante una aplicación online. Las características de la población apuntan hacia individuos con un nivel cultural alto, los cuales no presentan a priori ninguna dificultad para leer y comprender los enunciados del cuestionario y cuentan con un acceso normalizado a los dispositivos electrónicos.

Para la distribución del cuestionario se contó con la colaboración de Revista de Neurología. Ello se materializó en el contacto con las personas suscritas a las novedades de la revista, lo que se estimó 
Figura 1. Importancia de preguntar por las expectativas del encuentro clínico que tienen los pacientes.

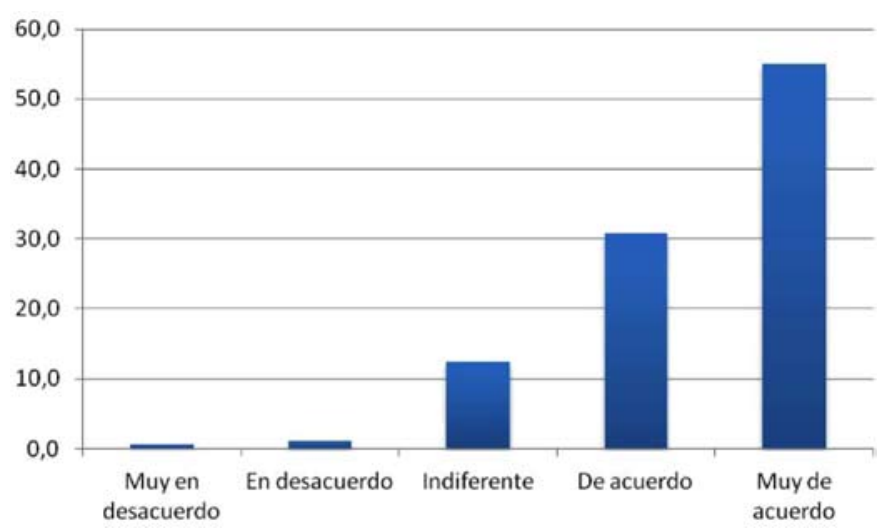

Figura 2. Un paciente que ha buscado información por su cuenta crea más problemas en el encuentro clínico que beneficios aporta.

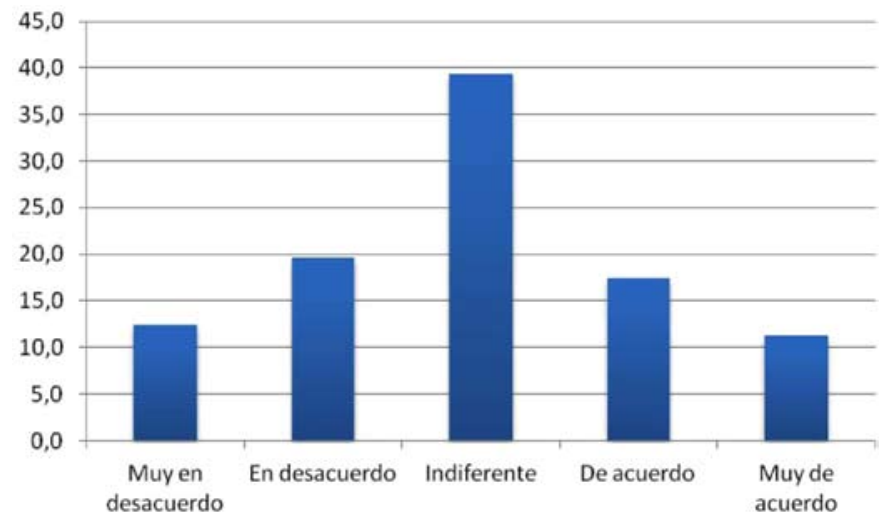

positivo para conseguir que el cuestionario llegase inicialmente a un amplio número de profesionales de la salud.

Antes de lanzar el cuestionario se realizó un pretest del $20 \%$ de la muestra para ajustarlo y comprobar si ofrecía dificultades o si inducía sesgos de respuesta.

\section{Resultados}

Respecto al sexo de las personas entrevistadas, las mujeres representan cerca del $70 \%$ del total. En relación a los grupos de edad, aproximadamente el $50 \%$ de los entrevistados tenían entre 40 y 59 años. Si atendemos a la categoría profesional, médicos y psicólogos son las dos categorías más representadas en la muestra. Por lo que respecta a los años de desarrollo de la profesión, estamos ante una muestra con una experiencia amplia, puesto que más del $80 \%$ cuenta con más de 10 años de ejercicio profesional. Respecto a la titularidad del centro de trabajo, un $44 \%$ de las personas entrevistadas ejercen en centros de titularidad pública, mientras que las personas que ejercen en centros de titularidad privada (concertados y no concertados) representan un $47 \%$.

Se presentan a continuación las distribuciones de frecuencias de las cuestiones referidas a las opiniones sobre el encuentro clínico y los pacientes. Los datos de las figuras expresan los porcentajes totales obtenidos en cada categoría en las distintas cuestiones.

La primera de las cuestiones planteadas, referida a la necesidad de contemplar las expectativas de los pacientes (Fig. 1), provoca un elevado grado de acuerdo: un $87 \%$ de las respuestas están de acuerdo o muy de acuerdo con esta afirmación. Se observa una circunstancia que va a poder apreciarse a lo largo de la exposición de los resultados: cuando las afirmaciones son muy directas en la formulación del trato hacia los pacientes, el grado de acuerdo es elevado; sin embargo, aquellas cuestiones que sitúan a los profesionales como los actores centrales del encuentro clínico también reciben un considerable apoyo. Se trata de una situación de aparente doble vínculo en la que, probablemente, desempeñen un papel importante los mensajes sociales predominantes, los cuales pueden entrar en cierta contradicción con los aspectos propios de las culturas profesionales.

En relación a la opinión de si los pacientes ven a los profesionales como responsables directos de la curación, las respuestas señalan que, de forma mayoritaria, los profesionales consideran que los pacientes les responsabilizan, lo que va en detrimento de la propia responsabilidad del paciente. Casi el $51 \%$ de las personas encuestadas se mostraron de acuerdo o muy de acuerdo con esta afirmación, mientras que un $35 \%$ se posicionaron en un punto intermedio y apenas un $14 \%$ se mostró en desacuerdo.

De manera congruente con la percepción a la que apunta la anterior cuestión, al plantear si se está de acuerdo con la afirmación 'a veces es mejor no ofrecer a los pacientes toda la información sobre su estado de salud', aunque existe una mayor dispersión de las respuestas, la mayoría se concentran especialmente en el desacuerdo con la afirmación propuesta (53\%), hay casi un $23 \%$ de entrevistados que están de acuerdo y un $24 \%$ no se decantan por el acuerdo o el desacuerdo. Esto apunta a una per- 
cepción favorable a la transparencia en el encuentro clínico y, por tanto, a la necesidad de que los pacientes se responsabilicen y cuenten con ello con la información suficiente.

No obstante, esta congruencia en las percepciones se difumina cuando se plantea la caracterización de los pacientes con información como problemáticos. La figura 2 muestra una distribución similar en las posiciones que están de acuerdo y en desacuerdo respecto a esta caracterización problemática de los pacientes informados. Destaca en esta cuestión la puntuación que obtiene la categoría 'indiferente', con algo más de un 39\% de respuestas.

En relación al rol del profesional en el encuentro clínico, existe un alto grado de acuerdo $(48,3 \%)$ en torno a la afirmación propuesta 'corresponde al profesional decidir cómo se va a desarrollar el encuentro clínico'. No obstante, es significativo que una frase tan categórica en relación al rol profesional reciba un considerable desacuerdo $(22,5 \%)$ y haya casi un tercio de encuestados que se muestren indiferentes.

En relación a la percepción sobre la búsqueda de información por parte de los pacientes, más de la mitad de las personas encuestadas se muestran en desacuerdo o muy en desacuerdo con la afirmación que planteaba que los pacientes deberían confiar en el conocimiento experto y no buscar otro tipo de información, aceptando la posibilidad de que accedan a fuentes alternativas de información. No obstante, el papel de internet en la conformación de las actitudes de la ciudadanía es visto mayoritariamente de forma negativa.

Sobre la conveniencia de tratar como iguales a los pacientes, un 67,4\% afirman estar de acuerdo o muy de acuerdo con la necesidad de un trato horizontal. De manera congruente, la necesidad de recabar las explicaciones que los propios pacientes dan a sus dolencias provoca un elevado acuerdo (80,3\%). Asimismo, el 82,6\% estuvieron de acuerdo en la importancia de preguntar al paciente por el uso de medicina complementaria o alternativa.

En la percepción sobre la confianza de los pacientes como base de la relación terapéutica destaca que, aun contando con un importante acuerdo alrededor de esta cuestión (64,6\%), casi el $23 \%$ de la muestra se manifiesta en desacuerdo. En este sentido, al plantear si la empatía es una cualidad secundaria, cerca del $85 \%$ estuvo en desacuerdo, lo que apunta a la empatía como una cualidad central.

Respecto a las percepciones sobre la competencia de los pacientes en distintos aspectos de la relación terapéutica, la figura 3 corrobora la existencia de una dispersión en las respuestas en la considera-
Figura 3. Los pacientes no tienen los conocimientos para identificar la etiología de su dolencia: esta es una tarea que compete a los profesionales.

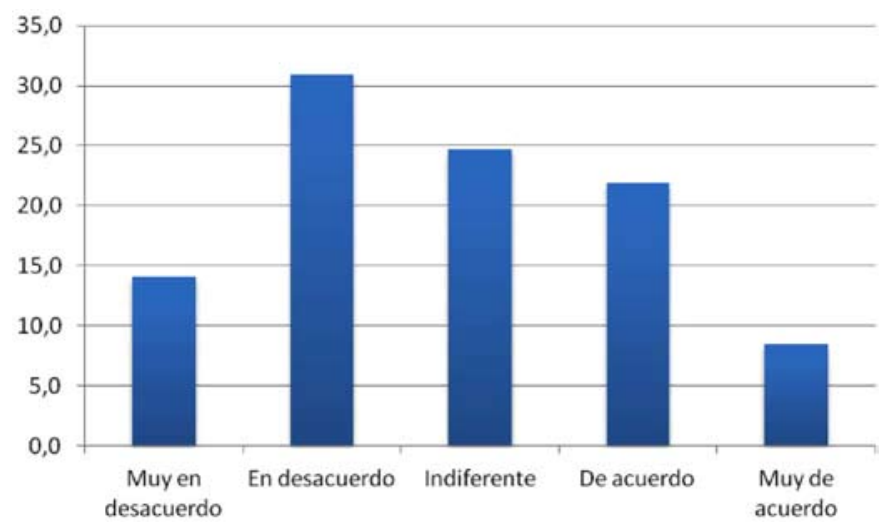

Figura 4. La población actual no quiere ni está preparada para coparticipar en la toma de decisiones sobre su salud.

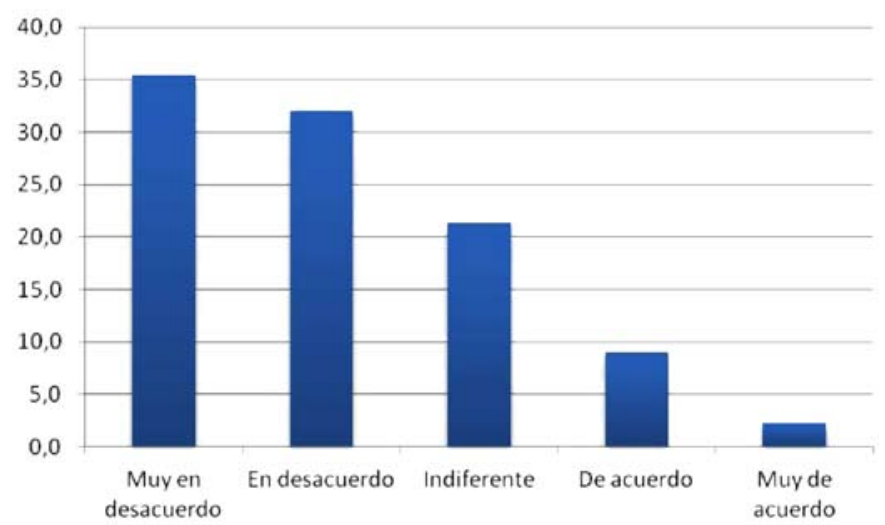

ción de la capacidad de los pacientes para identificar el origen de su dolencia. Un $45 \%$ se muestran contrarios a la afirmación recogida en la cuestión, mientras que casi un $31 \%$ están de acuerdo o muy de acuerdo. Destaca asimismo la existencia de casi un $25 \%$ de respuestas en la categoría 'indiferente'.

La siguiente cuestión ('un tratamiento no puede tener éxito si entra en conflicto con los valores o el estilo de vida del paciente') nos aproxima a la valoración de los posibles conflictos de los tratamientos con los valores y creencias de los pacientes. Un $87 \%$ se mostró de acuerdo o muy de acuerdo con esta afirmación. Por otra parte, casi el $72 \%$ no considera las cuestiones lingüísticas como imprescindibles para la relación terapéutica. 
Sobre la valoración que se tiene de los pacientes con más inclinación a preguntar y discutir las prescripciones, un $40 \%$ se muestra contrario a considerar como conflictivos a este tipo de pacientes. En el extremo opuesto se registra también un porcentaje de respuestas considerable: el $25 \%$ percibe como conflictivo al paciente que pregunta reiteradamente.

Los resultados recogidos en la figura 4 muestran un claro desacuerdo $(67,6 \%)$ con la consideración de la dejadez e incapacidad de la población para participar en la toma de decisiones sobre su salud. Estos resultados contrastan con el acuerdo registrado en relación a la afirmación de que los pacientes delegan la responsabilidad en los profesionales y no asumen su corresponsabilidad.

\section{Discusión}

El cuidado centrado en el paciente constituye hoy un término ampliamente utilizado en la práctica sociosanitaria, pero escasamente comprendido [16]. El ámbito discursivo propio de la interacción clínica reclama personalización e interactividad [6], características que, según se extrae de nuestros resultados, son todavía insuficientemente asumidas como consustanciales de los encuentros clínicos propios del siglo Xxi. Los resultados señalan la existencia de una posición mayoritaria más bien escéptica con las disposiciones culturales de os pacientes, la cual matizaría la importancia atribuida al papel que deberían desempeñar los pacientes en las relaciones en el ámbito sanitario.

Nuestros resultados coinciden con otros estudios que han interrogado por la participación de los pacientes en la toma de decisiones, como el de Ruiz et al, quienes concluyen que entre los profesionales se dan índices muy bajos de involucramiento de los pacientes en la toma de decisiones [12]. Esta tendencia se ha destacado incluso en el caso del colectivo de pacientes crónicos, donde sería esperable otro tipo de interacción más cooperativa y negociada. Sin embargo, no sólo las investigaciones empíricas [17], sino también algunas revisiones de material secundario, de las estrategias y programas destinados a la atención a la población de pacientes crónicos, detectan déficits en estos programas en cuanto a la incorporación de las decisiones compartidas en la relación clínica, la representación de los pacientes en los modelos de atención personalizada y la consideración de los factores sociales en la estratificación de los pacientes [18].

Volviendo a los resultados, cabe decir que cuando esta cuestión se plantea de manera directa, me- diante ítems como el de si los pacientes deberían ser tratados como iguales, recibe una valoración distinta: cuando el enunciado de las cuestiones formuladas a las personas entrevistadas apela al trato horizontal de manera explícita, el grado de acuerdo es mayor. Esta aparente discrepancia en las respuestas (centralidad del profesional y valoración del trato igualitario) podría indicar la existencia de dos marcos de interpretación en relativo conflicto: por un lado, la consideración de los pacientes desde una perspectiva escéptica en relación a sus disposiciones y capacidades, y por otro, la existencia de una serie de prescripciones que exhortan al trato horizontal.

Probablemente esta disonancia entre concepciones de la relación terapéutica es más fácil de identificar a través de los posicionamientos que tienen los individuos en función de su edad y de los años de desarrollo de la profesión. En los grupos de mayor edad y años de desarrollo está más presente la percepción relativamente crítica hacia los pacientes, mientras que entre los más jóvenes hay posiciones de distinto signo.

Es probable que las culturas profesionales tengan una importante influencia en la configuración de las percepciones sobre los procesos terapéuticos y el rol asignado a los pacientes. Esto se refleja en las diferencias observadas en las respuestas de las distintas categorías profesionales. Así, por ejemplo, profesionales de la medicina y personal de enfermería tienden a ubicarse en posiciones críticas con las capacidades y la predisposición de los pacientes en relación a su coparticipación en los procesos terapéuticos. Por su parte, especialistas en psicología y matrones/as son los más favorables a reconocer un mayor protagonismo a los pacientes en los encuentros clínicos y en los procesos terapéuticos. Entre los factores que pueden estar detrás de estas diferencias podría apuntarse el tipo de relaciones terapéuticas con los pacientes que cada categoría profesional ha construido históricamente.

En relación al sexo, la cuestión resulta más compleja de dilucidar. Hombres y mujeres tienen distintos grados de acuerdo y desacuerdo con las variables contempladas en este trabajo. Es probable que en este resultado se crucen varias dimensiones. Así, a lo ya apuntado en relación a la edad y los años de desarrollo de la profesión (que tendrían que ver con los procesos de socialización en los valores dominantes de las distintas profesiones), en el caso de la variable sexo es necesario pensar en la posibilidad de la existencia de valores vinculados con los roles de género, que podrían estar atravesando los posicionamientos de los y las profesionales. Estos resultados coinciden con los del estudio de Krupat et al [4]. 
Por último, y con la voluntad de señalar limitaciones del estudio, podríamos advertir un sesgo de la muestra que queda acotada a las personas suscritas a Revista de Neurología, a las cuales se les presupone un interés en participar en investigaciones que traten aspectos profesionales. Por otro lado, en un plano más metodológico, cabe decir que en el cuestionario diseñado por el equipo de investigación se han recogido las percepciones que tienen los profesionales sobre sus propios comportamientos y actitudes. Esta información, por tanto, no consigue retratar la reconversión de estas percepciones y concepciones en comportamiento real. Consideramos que las investigaciones que incluyen la observación directa como estrategia de recopilación de información (la clásica investigación de Atkinson [19] sería un ejemplo inspirador) podrían ofrecer un conocimiento de calidad sobre la disyuntiva entre lo que los profesionales dicen que hacen y lo que hacen realmente.

\section{Bibliografía}

1. Cockerham W. Sociología de la medicina. 8 ed. Madrid: Alhambra; 2010.

2. Seoane JA. La relación clínica en el siglo xxi: cuestiones médicas, éticas y jurídicas. Derecho y Salud 2008; 16: 1-28.

3. Seoane JA. Las autonomías del paciente. Dilemata 2010; 3: 61-75.

4. Krupat E, Rosenkranz SL, Yeager CM, Barnard K, Putnam SM, Inui TS. The practice orientations of physicians and patients: the effect of doctor-patient congruence on satisfaction. Patient Educ Couns 2000; 39: 49-59.

5. Pelzang R. Time to learn: understanding patient-centred care. Br J Nursing 2010; 19: 912-7.

6. Salvador-Liern V. Paraula i cultura de la salut: indagacions de lingüística mèdica. Caplletra: Revista Internacional de Filologia 2011; 50: 89-105.

7. Calvo F, Costa AM, García-Conde J, Megía M.
Sin reconocimiento recíproco no hay calidad asistencial. Rev Esp Salud Publica 2011; 85: 459-68.

8. Cófreces P, Ofman SD, Stefani D. La comunicación en la relación médico-paciente. Análisis de la literatura científica entre 1990 y 2010. Revista de Comunicación y Salud 2014; 4: 19-34.

9. Barca I, Parejo R, Gutiérrez P, Fernández F, Alejandre G, López de Castro F. La información al paciente y su participación en la toma de decisiones clínicas. Aten Primaria 2004; 33: 361-5.

10. Marín-Torres V, Valverde-Aliaga J, Sánchez-Miró I, Sáenz del Castillo-Vicente MI, Polentinos-Castro E, Garrido-Barral A. Internet como fuente de información sobre salud en pacientes de atención primaria y su influencia en la relación médicopaciente. Aten Primaria 2013; 45: 46-53.

11. Mira JJ, Llinás G, Lorenzo S, Aibar C. Uso de internet por médicos de primaria y hospitales y percepción de cómo influye en su relación con los pacientes. Aten Primaria 2009; 41: 308-14.

12. Ruiz R, Peralta L, Pérula LA, Gavilán E, Loaissa JR. Participación del paciente en la toma de decisiones en atención primaria: una herramienta para su medición. Aten Primaria 2010; 42: 257-63.

13. Dwamena F, Holmes-Rovner M, Gaulden CM, Jorgenson S, Sadigh G, Sikorskii A, et al. Interventions for providers to promote a patient-centred approach in clinical consultations. Cochrane Database Syst Rev 2012; 12: CD003267.

14. Ferguson WJ, Candib LM. Culture, language, and the doctorpatient relationship. FMCH Publications and Presentations 2002; 61: 353-61.

15. Weech-Maldonado R, Carle A, Weidmer B, Hurtado M, NgoMetzger Q, Hays RD. The Consumer Assessment of Healthcare Providers and Systems (CAHPS ${ }^{\circ}$ ) cultural competence item set. Med Care 2012; 50 (Suppl 2): S22-31.

16. Stewart M. Towards a global definition of patient centred care. BMJ 2001; 322: 444-5.

17. Danet A, Escudero, MJ, Prieto MA, March JC. Comunicación paciente-médico y toma de decisiones en salud en Andalucía. Expectativas de pacientes crónicos del Sistema Sanitario Público Andaluz. Revista de Comunicación y Salud 2012; 2: 247-57.

18. Coronado-Vázquez V, Gómez-Salgado J, Cerezo-Espinosa de los Monteros J, Canet-Fajas C, Magallón-Botaya R. Equidad y autonomía del paciente en las estrategias de atención a personas con enfermedades crónicas en los servicios de salud de España. Gac Sanit 2018; Jul 28. [Epub ahead of print].

19. Atkinson P. Medical talk and medical work. A liturgy of the clinic. London: Sage; 1995. 